\title{
REGION, the new OPEN ACCESS ERSA journal
}

\author{
Jouke van Dijk ${ }^{1}$, President of ERSA \\ ${ }^{1}$ University of Groningen, Groningen, The Netherlands (email: jouke.van.dijk@rug.nl)
}

Received: 12 June 2014/Accepted: 12 June 2014

In recent years, regional science has seen an increase in research output, participants and papers presented at the annual ERSA conferences and in initiatives for organizing international workshops and activities by ERSA. For the future success of these activities, it is important that ERSA has an open eye for new developments in publication outlets. Currently there is a growing belief that scientific knowledge should be available for free to everyone because the research is often already paid for by taxpayers. Following this logic, more and more national and international funding organizations require open access publication of research output of the projects they fund. The European Commission, for example, announced that all publications arising from its Horizon 2020 funding programme must be open access. Thus, ERSA took the initiative to create a new online and fully open access journal. A great opportunity to realize this idea came up when Gunther Maier suggested that ERSA could apply in cooperation with his university, the Vienna University of Economics and Business (WU), for the Initial Funding Program for Open Access Journals of the Austrian Science Fund (FWF). In close cooperation with Gunther Maier, ERSA applied with the proposal to launch the new online and fully open access journal REGION as the official scientific journal of ERSA, which was selected for funding.

Therefore, as the president of ERSA, I am very pleased to announce the launch of the new online and fully open access (DIAMOND-status) journal REGION as the official scientific journal of ERSA. DIAMOND open access is the highest and most preferred open access status, because it is freely accessible to all readers, there is no submission or publication fee for authors, it is inclusive of peer review and REGION hosts the final version of the article. REGION aims to strengthen ERSAs role in facilitating the free dissemination and creation of high-quality research on issues in regional science. Although ERSA is based in Europe, it is meant to be an association that is globally relevant. This goal fits the open access nature of the journal. At the same time, the link to ERSA as a learned organization and the resources this offers gives the journal every chance of developing into a high-quality and well-read journal in regional science.

REGION accepts traditional research articles. In addition, it welcomes contributions that focus on the collection, analysis and visualization of regional data. Such contributions are hosted in the Resources section. Importantly, the journal also includes a young scholar section targeted at the newest generation of researchers in regional science. The section can accommodate PhD-work, but it also offers a suitable outlet for outstanding undergraduate research work. ERSA actively seeks to stimulate and train researchers new to the field. As part of this effort, ERSA offers a yearly summer school on the current state-of-the-art in regional science. In addition, the successful EPAINOS-sessions at the annual ERSA conference offer young researchers the opportunity to present their work to a large audience. The Young Scholar Section in REGION is a useful addition to the existing activities conducted under the umbrella of ERSA.

The journal is in the hands of a very capable and enthusiastic editorial team that will ensure the quality of the articles and run the journal on a day-to-day basis. We thank 
Gunther Maier and the Vienna University of Economics and Business for their support in writing the proposal and for funding and handling the technical infrastructure of REGION. Finally, the journal is supported by ERSA in matters of promotion. Even though the journal is in good hands, its eventual success will depend on your inputs, on the contribution of the ERSA community and the regional science community as a whole. As an open access and online journal, REGION stands out precisely because it can rely on a large community of high quality researchers organized in ERSA. Therefore, I ask you to invest some time if you are asked as a reviewer, to think about REGION as an outlet for your work and frequent its website to check the latest publications.

With your help, I have great confidence that REGION, as the flagship journal of ERSA with Diamond open access status, will grow to be a highly-visible and highquality journal in regional science. By removing all financial barriers, ERSA intends to stimulate the dialog in the regional science community and make REGION the medium for cutting edge research findings on regional issues for scientists, policy makers, NGOs and the general public at the global scale. 